\title{
Les myiases des artiodactyles sauvages et des proboscidiens d'Afrique centrale
}

\author{
par M. GRABER $\left({ }^{*}\right)$ et J. THAL $\left({ }^{* *}\right)$
}

\begin{abstract}
RÉSUME
Les auteurs font l'inventaire des larves d'Oestridae et de Gasterophilidae existant en Afrique centrale chez les Artiodactyles sauvages et chez les éléphants. Huit espèces différentes ont été dénombrées dont deux nouvelles pour le Tchad et pour la R. C. A. : Rhinoestrus phacochoeri du phacochère et Strobiloestrus clarkii du Reedbuck. Les mammifères les plus atteints sont l'éléphant, le damalisque, le bubale et le phacochère. Dans ce cas, les myiases sont essentiellement cavitaires (sinus, cavités nasales, estomac). Il existe également chez la Gazelle dorcas et le Reedbuck des myiases cutanées avec localisation des parasites sous la peau du dos.
\end{abstract}

\section{INTRODUCTION}

Les myiases sont des affections liées à la présence en différents points du corps de larves de Diptères (Brachycera) appartenant surtout aux famille des Oestridae et des Gasterophilidae.

En Afrique tropicale, elles sont fréquemment rencontrées chez les animaux domestiques et chez les animaux sauvages, notamment au Tchad où un premier bilan a été dressé, il y a déjà une quinzaine d'années (4).

Depuis, d'autres recherches menées dans le cadre d'une étude sur la pathologie de la faune sauvage dans l'Est de la République Centrafricaine (R. C. A.) (11) ont permis de compléter cet inventaire et de l'étendre à toute l'Afrique centrale.

\section{MATÉRIEL ET MÉTHODE}

En R. C. A., 196 Artiodactyles et Proboscidiens ont été autopsiés entre 1969 et 1972 : Phacochoerus aethiopicus, Pallas, le phacochère: 73.

(*) Service de parasitologie, Ecole Nationale Vétérinaire de Lyon, Marcy l'Etoile, 69260, Charbonnières-lesBains.

$(* *)$ I. E. M. V. T. 10 , rue Pierre-Curie, 94700, Maisons-Alfort.
Potamochoerus porcus, Linné, le potamochère : 2.

Hylochoerus meinertzageni, Thomas, l'hylochère : 3.

Bubalus (Syncerus) caffer, Sparrman, le buffle : 71.

Taurotragus derbianus, Gray, l'éland de Derby: 3. Tragelaphus scriptus, Pallas, le guib : 3 .

Alcelaphus lelwel, Heuglin, le bubale : 13.

Damaliscus korrigum, Ogilby, le damalisque : 1. Hippotragus equinus, Desmarest, l'hippotrague : 7.

Kobus defassa, Rüppel, le cob defassa : 7.

Adenota cob, Erxleben, le cob de Buffon: 5.

Redunca redunca nigeriensis, Blaine, le reedbuck : 2 .

Sylvicapra grimmia, Linné, le céphalophe couronné : 3.

Ourebia ourebi, Zimmerman, l'ourébi : 3.

Loxodonta africana, Blumenbach, l'éléphant d'Afrique : 3.

A cette liste, il faut ajouter un phacochère, trois buffles, un bubale, deux ourébis, un guib et un éléphant abattus en 1962-1963 autour de Bambari et de Birao.

Les parasites ont été déterminés en se basant sur l'excellente monographie de ZUMPT (12). 


\section{LES PARASITES}

En R. C. A., ont été identıfiées les larves suivantes :

\section{DES GASTEROPHILIDAE}

1.1. Cobboldia loxodontis Brauer, 1897 (Cobboldiinae)

Localisation et hôte : estomac de l'éléphant. Origines: $228 \mathrm{~B} \mathrm{~b}\left(^{*}\right)$ (Dinga); $255 \mathrm{Dd}$; $246 \mathrm{~B} \mathrm{~d}$.

Cobboldia loxodontis est un parasite classique de l'éléphant $(3,12)$ que l'on retrouve dans toutes les régions d'Afrique tropicale où vit cet animal, c'est-à-dire de la Côte-d'Ivoire à l'Afrique du sud et de la République populaire du Congo à l'Ethiopie (3). Il est très répandu au Tchad (4).

Récemment, ZUMPT et WETZEL (14) ont montré que les larves III implantées dans la muqueuse stomacale ou libies à l'intérieur de l'organe le quittent au bout d'un temps variable et sont expulsées par la voie buccale. Elles tombent sur le sol, s'y enfoncent et se transforment en pupes. Celles-ci donnent naissance, au bout de 2 à 3 semaines, à 2 types d'imago :

— une mouche bleue de 10 à $14 \mathrm{~mm}$, Cobboldia loxodontis, aplatie dorso-ventralement et dont le front fait fortement saillie. La tête est orange, l'abdomen et le thorax d'un bleu métallique uniforme. Les pattes sont d'un noir luisant et les ailes légèrement enfumées;

- une mouche verte de 11-13,5 mm, Cobboldia chrysidiformis, recueillie dans le haut Uélé (Zaïre) - région limitrophe du Soudan et de la R. C. A. dans le nord-est du Zaïre - par RODHAIN et BEQUAERT (9) et revue depuis en Afrique du sud (14).

La moitié antérieure de la tête est brun rougeâtre et la moitié postérieure est bleue. Le thorax et l'abdomen sont d'un vert métallique à reflets bleuâtres ou violacés. Les pattes sont bleues et les ailes d'une couleur tirant sur le brun jaunâtre.

Les larves stomacales issues de ces 2 espèces de Cobboldia qui peuvent d'ailleurs cohabiter chez le même animal (9) sont rigoureusement semblables et aucun caractère morphologique particulier ne permet de les différencier l'une de l'autre.

(*) Coordonnées I. B. A. H. qui utilisent des carrés délimités par les latitudes et par les longitudes.
Au Tchad, des mouches bleues de Cobboldia loxodontis ont été obtenues après mise en élevage de plusieurs lots d'origine diverse. Malheureusement, ils n'ont pu être tous strictement contrôlés, soit parce que les parasites avaient été formolés, soit parce que les élevages sur sable sont demeurés improductifs.

Dans ces conditions, il est impossible d'affirmer actuellement que Cobboldia loxodontis est la seule espèce de Gasterophilidae de l'éléphant existant en Afrique centrale.

La $3^{\text {e }}$ espèce de Cobboldia de l'éléphant d'Afrique, Cobboldia roverei Gedoelst, 1915 n'est connue que par ses larves au stade $l$ et par ses larves au stade III. Ces dernières sont pourvues de papilles coniques insérées sur les bourrelets latéraux des segments 7 à 11, papilles qui sont absentes chez les autres Cobboldia.

Ce parasite n'existe qu'au Zaïre $(1,14)$ et, jusqu'à maintenant, sa présence n'a jamais été signalée dans d'autres pays d'Afrique, notamment au Tchad et en R. C. A.

\subsection{Neocuterebra squamosa Grünberg, 1906 (Neocuterebrinae)}

Localisation et hôte : éléphant. Larves dans le tissu élastique $\mathrm{du}$ coussinet plantaire qu'elles entament profondément en traversant la sole pédieuse.

Origine : est de la R. C. A.

L'aire de répartition de Neocuterebra squamosa couvre donc le Cameroun (12), la R. C. A. et le nord-est du Zaïre $(6,9)$.

\section{OESTRIDAE}

\subsection{Kirkioetrus minutus Rodhain et Bequaert,} 1915 (Oestrinae)

Localisation et hôte : sinus et cavités nasales du bubale.

Origine: Bahr Aouk et Région du Lac Mamoun $(229 \mathrm{Cd})$, à la frontière du Tchad et de la R. C. A. (En R. C. A., les bubales sont porteurs d'un grand nombre de larves d'Oestridés (11). Malheureusement, 2 prélèvements seulement ont pu être examinés dans leur totalité. L'inventaire est donc loin d'être complet.)

Cet Oestridé est un parasite commun des antilopes africaines (gnou, bubale, damalisque) en Afrique de l'est, en Afrique du sud, ainsi qu'au Zaïre, dans l'Uélé (7). 


\subsection{Kirkioestrus blanchardi Gedoelst, 1914 (Oestrinae)}

Localisation et hôte : sinus du bubale.

Origine : Bahr Aouk et Lac Mamoun.

Kirkioestrus blanchardi affecte surtout les bubales, plus rarement les damalisques de l'Afrique de l'Ouest (Côte-d'Ivoire), d'Afrique de l'est (Mozambique) et d'Afrique centrale (Tchad, R. C. A., nord-est du Zaïre).

\subsection{Rhinoestrus phacochoeri Rodhain et Be- quaert, 1915 (Oestrinae)}

Localisation et hôte : sinus du phacochère.

Origine : toute la zone cygénétique de l'est de la R. C. A.

Les larves au stade III sont caractéristiques. Elles mesurent de 11 à $17 \mathrm{~mm} \times 5-7 \mathrm{~mm}$ (moyenne 16,5 $\times 5,5 \mathrm{~mm}$ ). Les plus jeunes sont de couleur blanc jaunâtre. Cette coloration change au fur et à mesure que les larves vieillissent et les plus âgées sont sombres avec des bandes noires irrégulièrement disposées.

La face dorsale de la larve est complètement nue.

La face ventrale porte des épines qui sont réparties de la façon suivante : 1 ou 2 rangées sur les segments 3 et $4 ; 3$ rangées du $5^{\mathrm{e}}$ au $11^{\mathrm{e}}$ segment. Le segment céphalique et le dernier segment (le $12^{\mathrm{e}}$ ) sont inermes. Les péritrèmes sont percés d'un grand nombre de pores et le hile est relativement large.

Les larves au stade II se distinguent des précédentes par leur taille $(8,5-10 \times 2-4 \mathrm{~mm}$; moyenne $8,9 \times 3,2 \mathrm{~mm}$ ) et par l'armature de la face ventrale qui ressemble à celle de la larve III, sauf au niveau du $11^{\mathrm{e}}$ et du $12^{\mathrm{e}}$ segments qui sont ornés respectivement de 4 et de 5 rangées d'épines.

Les larves II et les larves III sont souvent mélangées chez le même animal.

Rhinoestrus phacochoeri paraît strictement localisé à l'Afrique centrale : nord-est du Zaïre (7, 8), Cameroun (12), est de la R. C. A. et Tchad (zone d'Ouabang, moyenne vallée du Chari, 1965). Jusqu'à plus ample informé, il ne semble pas exister ailleurs.

\subsection{Oestrus variolosus Loew, 1863 (Oestrinae)} Localisation et hôte : sinus du damalisque. Origine : $218 \mathrm{D}$ a (Rivière Gounda).

Cet Oestriné de couleur sombre et fortement aplati mesure $18-20 \times 9,5-10 \mathrm{~mm}$. La face dor- sale est totalement nue. Les segments de la face ventrale sont couverts d'épines, très petites à la base du segment 2 et beaucoup plus longues au fur et à mesure que l'on se rapproche de l'extrémité postérieure. Elles sont disposées en rangées complètes (sauf sur le $3^{\mathrm{e}}$ segment), à raison de 3 à 6 , du segment 4 au segment 10 . Les 2 derniers n'en possèdent que 2 . Les péritrèmes poreux sont entièrement clos.

Oestrus variolosus est connu dans toute l'Afrique au sud du Sahara, surtout chez les Alcelaphinae et chez les Hippotraginae (4).

\subsection{Gedoelstia cristata Rodhain et Bequaert, 1913 (Oestrinae)}

Localisation et hôte : sinus et cavités nasales du bubale.

Origine : Bahr Aouk et Lac Mamoun.

Cette espèce est souvent associée à Kirkioestrus minutus et à Kirkioestrus blanchardi. Elle a les mêmes hôtes et la même répartition géographique qu'Oestrus variolosus.

\subsection{Strobiloestrus clarkii Clark, 1841 (Hypo- derminae)}

Localisation et hôte : reedbuck. Conjonctif sous-cutané du dos (reins).

Origine: Lac Mamoun (21 $10 \mathrm{~B} \mathrm{~b}$ ).

Dix-huit larves au stade II ont été extraites des nodules cutanés. De couleur blanc ivoire, elles mesurent $9-12 \times 5-8 \mathrm{~mm}$ (moyenne $10,2 \times 7,2 \mathrm{~mm})$. Chaque larve comporte $12 \mathrm{seg}-$ ments. Les premiers (du $3^{\mathrm{e}}$ au $6^{\mathrm{e}}$ ) portent des protubérances lobulaires, vastes excroissances qui donnent à cet Hypoderminé un aspect très particulier. Sur le $5^{\mathbf{e}}$ et sur le $6^{\mathrm{e}}$ segments, on en compte 6. Au-delà, elles disparaissent totalement et la larve s'amincit progressivement. Les péritrèmes renferment 15 pores.

En Afrique au sud du Sahara, 2 autres espèces de Strobiloestrus ont été identifiées:

- Strobiloestrus vanzyli Zumpt, 1961 en Zambie $(5,12)$.

- Strobiloestrus eriksoni Poppius, 1907 au Katanga (9).

Elles paraissent strictement inféodées au Lechwe (Kobus leche).

Strobiloestrus clarkii qui, jusqu'à présent n'a été rencontré qu'en Afrique du sud (12) infeste un plus grand nombre d'herbivores : oreotrague, steenbok, rhebok, kudu et reedbuck (Redunca arundinum). 
Il est difficile d'identifier exactement le parasite en cause, car les larves II et III des 3 èspèces connues présentent des caractères morphologiques semblables. Toutefois, compte tenu de la spécificité d'hôte qui, en ce qui concerne Strobiloestrus vanzyli et Strobiloestrus eriksoni, paraît très étroite, les parasites du Redunca de R. C. A. - comme ceux du reedbuck du Natal $(12,13)$ - semblent devoir être rapportés à Strobiloestrus clarkii. En attendant d'autres renseignements, ils seront donc provisoirement assimilés à cette espèce.

Outre la R. C. A. et l'Afrique du sud, cet Hypoderminé a été recueilli en 1965 sous la peau du dos d'un reedbuck (Redunca redunca nigeriensis) tué à Balekoutou dans le centre du Tchad. C'est donc la seconde fois qu'un Strobiloestrus est mis en évidence au nord de l'équateur.

\section{COMMENTAIRES}

D'une façon générale, en Afrique centrale (Tchad, R. C. A., Nord Cameroun).

1) Sur environ 390 Artiodactyles et Proboscidiens autopsiés entre 1954 et 1972, les espèces suivantes se sont révélées indemnes de toute myiase, qu'elle soit cavitaire ou cutanée : potamochère $(2)(*)$, hylochère $(3)$, hippopotame (3), buffle (88), éland de Derby (4), guib (6), hippotrague (20), cob defassa (25), cob de Buffon (20), céphalophe couronné (7), ourébi (13), gazelle corinne (21), gazelle dama (9), oryx (9), addax (1), grand koudou (2).

Le même phénomène a été observé en Tanzanie dans le Parc de Serengeti (10).

2) Les agents des myiases cavitaires (Oestrinae et Cobboldiinae) affectent :

\begin{tabular}{|c|c|c|c|}
\hline \multirow{2}{*}{$\begin{array}{c}\text { Nombre d'animaux } \\
\text { autopsiës et espèces }\end{array}$} & \multicolumn{3}{|c|}{ Nombre d'animaux parasités et pourcentage d'infestation } \\
\hline & $T \circ t$ a 1 & Tchad et Cameroun & R. C. A. \\
\hline E1êphant : 17 & $\begin{array}{l}16 \text { sur } 17 \\
(95 \mathrm{p} .100)\end{array}$ & 13 sur 13 & 3 sur 4 \\
\hline Damalisque : 13 & $6 \operatorname{sur} 13$ & 5 sur 12 & 1 sur 1 \\
\hline Bubale : 34 & $\begin{array}{l}13 \text { sur } 34 \\
(38 \text { p. } 100)\end{array}$ & 2 sur 20 & 11 sur 14 \\
\hline Phacochère : 92 & & $\begin{array}{l}\text { Les renseignements } \\
\text { font dêfaut. }\end{array}$ & $\begin{array}{l}\text { de } 25 \text { à } 40 \text { p. } 100 \\
\text { selon les régions }\end{array}$ \\
\hline
\end{tabular}

Comme dans bien d'autres régions d'Afrique tropicale (10), le bubale, le damalisque et l'éléphant sont les animaux les plus atteints. Le phacochère semble également entrer dans cette catégorie. Cependant, l'ouverture des têtes n'a pas été effectuée de façon systématique, ce qui ne permet pas de donner des pourcentages précis.

3) Les myiases cutanées des herbivores sauvages sont dues à 2 Hypoderminae:

- au Tchad et en R. C. A., Strobiloestrus clarkii qui affecte essentiellement le reedbuck (2 Redunca parasités sur les 10 autopsiés).

- au Tchad, Przhevalskiana corinnae Crivelli, 1892 parasite de la gazelle dorcas au nord du $13^{\mathrm{e}}$ parallèle (4). Sur les 36 gazelles examinées, 16 d'entre elles (soit 44 p. 100) présentaient des nodules localisés à la peau du dos, des épaules et du périnée.

Il est bon de rappeler qu'en Afrique centrale, si les myiases cutanées des herbivores sauvages sont assez répandues, il n'en est pas de même chez les ruminants domestiques et aucun cas d'hypodermose n'a, jusqu'à maintenant, été observé ni chez le zébu ni chez le mouton, ni chez la chèvre.

4) Ces myiases, qu'elles soient cutanées ou cavitaires, sont, la plupart du temps, bien supportées car le niveau de l'infestation est faible : de 5 à 30 parasites par animal chez les artiodactyles; de 50 à plus de 1000 chez l'éléphant.

5) En matière d'épidémiologie, on ne possède que peu de renseignements. Selon ZUMPT (12), les larves de Cobboldia loxodontis, de Kirkioestrus minutus et d'Oetrus variolosus se rencontrent toute l'année à différents stades de développement, ce qui a été vérifié au Tchad et en R. C. A., tout au moins pour les Cobboldia de l'éléphant.

Il semble en être de même pour Przhevalskiana corinnae : en effet, dans le nord du Tchad, des

(*) Nombre d'autopsies. 
larves I, II et III dans des proportions variables ont été récoltées au début de la saison des pluies (juillet), au début et au cœur de la saison sèche (octobre et décembre).

\section{CONCLUSIONS}

L'examen d'une collection de parasites rassemblée en R. C. A. entre 1969 et 1972 a permis de compléter les renseignements que l'on possédait déjà en Afrique centrale sur les larves d'Oestridae et de Gasterophilidae susceptibles de provoquer des myiases chez les artiodactyles sauvages et chez les éléphants.

Huit espèces différentes ont été dénombrées. Deux d'entre elles sont signalées pour la pre- mière fois au Tchad et en R. C. A. : Rhinoestrus phacochoeri du phacochère et Strobiloestrus clarkii du reedbuck.

Deux types de larves ont été rencontrées: - Des larves cavitaires d'Oestrinae et de Cobboldiinae localisées aux sinus, aux cavités nasales et à l'estomac. Par ordre d'importance, elles affectent l'éléphant, le damalisque, le bubale et le phacochère.

- Des larves cutanées d'Hypoderminae enfouies sous la peau du dos des gazelles dorcas et du reedbuck.

Les autres animaux sauvages (16 espèces au total) ne sont pas atteints.

Ces myiases paraissent, en général, bien supportées.

\section{SUMMARY}

\section{Myiasis of artiodactyla and proboscidea in Central Africa}

In Central Africa (Chad, Centrafrican Republic, North Cameroon), the authors draw up inventory of Oestridae and Gasterophilidae larvae present in wild artiodactyla and african elephants.

Eight different species were counted of which two are mentionned for the first time in Chad and R. C. A. : Rhinoestrus phacochoeri in warthog and Strobiloestrus clarkii in reedbuck.

Internal larvae were recovered only from the nasal cavities and frontal sinuses of hartebeest, topi, warthog (Oestrinae) and from stomach of african elephant (Cobboldiinae).

External larvae (Hypoderminae) were found in boils located on the back of Dorcas gazelle and reedbuck.

Other mammals ( 12 species) were not affected.

Generally, myiasis in wild animals seem well tolerated.

\section{RESUMEN}

Las miasis de los artiodíctilos salvajes y de los proboscidios de Africa Central

Los autores hacen el inventario de las laryas de Oestridae y de Gasterophilidae existentes en los artiodáctilos salvajes y en los elefantes de la Africa Central.

Se empadronaron ocho especies diferentes de las cuales dos nuevas en el Chad y en la República Centroafricana Rhinoesirus phacochoeri del facoquero y Strobiloestrus clarkii del reedbuck.

Los mamíferos más atacados son el elefante, el damalisco, el bubalo y el facoquero. En este caso, las miasis son sobretodo cavernosas (senos, cavidades nasales, estómago).

Existen tambièn en la gacela dorcas y el reedbuck miasis cutáneas con localización de los parásitos bajo la piel del lomo.

\section{BIBLIOGRAPHIE}

1. GEDOELST (L.). Notes sur les Oestridés. 1. Revue Zool. afr., $1915(a), 4(2)$ : 144-161.

2. GEDOELST (L.). Notes sur les Oestridés. 2. Revue Zool. afr., $1915(b), 4(2)$ : 259-264.

3. GEDOELST (L.). Les trois stades larvaires de Cobboldia loxodontis. Annls. Parasit. hum. comp., 1923,1 (4) : 354-362.
4. GRABER (M.), GRUVEL (J.). Etude des agents des myiases des animaux domestiques et sauvages d'Afrıque équatoriale. Rev. Elev. Méd. vét. Pays trop., 1964, 17 (3) : 535-554.

5. HOWARD ( $G$. W.). Differentially delayed development in the Lechwe warble Strobiloestrus vanzyli (Diptera: Oestridae). Parasitology, 1975, 71 (2) : X. 
6. RODHAIN (J.). Contribution à la faune des Oestridés du Congo belge. Annls. Parasit. hum. comp., 1927, 5 (3) : 193-213.

7. RODHAIN (J.), BEQUAERT (J.). Sur quelques Oestridés du Congo (Communication préliminaire) Bull. Soc, Path. exot., 1915(a), 8 (7) : 452-458.

8. RODHAIN (J.), BEQUAERT (J.). Sur quelques Oestridés du Congo (Deuxième communication préliminaire). Bull. Soc. Path. exot., 1915(b), 8 (9) : $687-695$.

9. RODHAIN (J.), BEQUAERT (J.). Sur quelques Oestridés du Congo (troisième communication préliminaire). Bull. Soc. Path. exot., 1915(c), 8 (10) : 765-778.

10. SACHS (R.), Uner den befall ostrafrikanischer
Wildtiere mit parasitischen fliegenlarven (Diptera, Oestridae). Acta trop., 1970, 27 (4) : 281-290.

11. THAL (J.). Les maladies similaires à la peste bovine. Etude et lutte. Projet P. N. U. D./C. A. F. 13, Maisons-Alfort, I. E. M. V. T., 1972, 57 p.

12. ZUMPT (F.). Myiasis in man and animals in the world. London, Butterworths, 1965(a), $267 \mathrm{p}$.

13. ZUMPT (F.), STIMIC (M.). Strobiloestrus clarkii (Clark) reared for the first time from the african Steenbok, Rhaphicerus campestris. Z. Parasitkde., 1965(b), 25 : 339-341.

14. ZUMPT (F.), WETZEL (H.). Fly parasites (Diptera : Oestridae and Gasterophilidae) of the african elephant, Loxodonta africa (Blumenbach) and their problems. Koedoe, 1970, 13 : 109-121. 